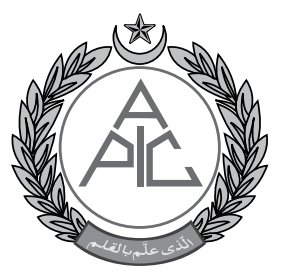

\title{
Our experience of submental intubation: a reliable alternative to elective tracheostomy in maxillofacial trauma
}

\author{
Uzair Luqman, BDS, FCPS, FFDRCSI, FAOCMF', \\ Muhammad Umar Qayyum, BDS, FCPS, FFDRCSI², Shahida \\ Tasneem, MBBS, MCPS ${ }^{3}$, Kaleem Ullah, BDS, FCPS ${ }^{1}$, Mohsin Khan, \\ BDS, MCPS ${ }^{4}$, Jahangir Khan, BDS ${ }^{4}$
}

\begin{abstract}
Objective: The aim of this study was to evaluate retrospectively the frequency, indications, and outcome of submental intubation in maxillofacial trauma patients.

Methodology: This retrospective study was performed at our maxillofacial surgery department. Retrospective data were collected from electronic patient records and patients' operation notes from 1st June 2013 to 30th April 2018. All patients who had submental intubation performed irrespective of age and gender were included. Indications, intraoperative / post-operative course, any complications and esthetic results were evaluated.

Results: During the period of study from 1st June 2013 to 30th April 2018, a total of 253 patients were operated under general anesthesia for various maxillofacial injuries at our hospital. Out of 253 operated patients, 185(73.1\%) were males and 68(26.9\%) were females with mean age of 32.7 years. A total of 7 submental intubations were performed. Out of these, 2 patients had panfacial trauma, 3 had comminuted Lefort II and III fractures and 2 had comminuted nasoorbitoethmoid (NOE)/ frontal complex fractures. No intraoperative complications were recorded. Postoperatively, one patient had persistent orocutaneous fistula with salivary leakage which was managed conservatively and healed within 4 weeks after surgery.
\end{abstract}

${ }^{1}$ Consultant Oral and Maxillofacial Surgeon, KRL General Hospital, Islamabad (Pakistan)

${ }^{2}$ Consultant Oral and Maxillofacial Surgeon, $\mathrm{CMH}$ Kharian Cantt, (Pakistan) ${ }^{3}$ Head of Anesthesiology, KRL General Hospital Islamabad, (Pakistan)

${ }^{4}$ Resident Oral and Maxillofacial Surgeon, KRL General Hospital Islamabad, (Pakistan)

\section{Correspondence:}

Uzair Luqman, H. No. 76A, Street 4, Phase 2, Bahria Town Islamabad, (Pakistan); Phone: +921-321-5080354; E-mail: uzair.luqman@gmail.com

Received: 18 Oct 2018

Reviewed: 20 Oct 2018

Accepted: 30 Oct 2018

Conclusions: Submental intubation is a simple, safe and fast alternative to tracheostomy for operative airway management in maxillofacial trauma patients.

Citation: Luqman U, Qayyum MU, Tasneem S, Ullah K, Khan M, Khan J. Our experience of submental intubation: a reliable alternative to elective tracheostomy in maxillofacial trauma patients. Anaesth Pain \& Intensive Care 2018;22 Suppl 1:S113-S117

\section{INTRODUCTION}

Maxillofacial trauma management under general anesthesia is challenging both to the maxillofacial surgeon as well as the anesthesiologist. Extensive maxillofacial injuries may lead to derangement of the architecture and disruption of different components (soft tissues, bone, and cartilaginous) of the upper airway, often with little external evidence of the deformity. ${ }^{1}$ They pose a big challenge to the anesthesiologist to secure the airway and safely intubate these patients. From the maxillofacial surgeon's perspective, treatment requires an unhindered access to the whole maxillofacial area. This leads to both the specialties sharing the same 
area to accomplish their management goals. On one hand the anesthesiologist has to set a priority to maintain a stable airway, and on the other, the surgeon requires an unhindered access to the maxillofacial area for adequate exposure and surgery. ${ }^{2}$ Recent advances in trauma management have made open reduction and internal fixation a norm. The oral or nasal endotracheal tubes becomes a major hindrance for the surgeons in such cases. The surgeon may also need to utilize intraoperative intermaxillary fixation to assess proper occlusion, which may take the most practiced route of intubation (oral intubation) off the shelf. ${ }^{1}$

Different methods and combinations have been tried over the years for such surgical endeavors, including staged technique which requires switching from oral to nasal route while treating the occlusal segment of the facial skeleton. ${ }^{2}$ A disadvantage of nasal intubation in severe maxillofacial trauma is the fracture of the base of skull, which may risk tube entry into the anterior cranial fossa causing brain damage, CSF leak, and possibly meningitis. ${ }^{3}$ It may also be difficult or even contradicted in cervical spine injuries. Also, the switch over is time consuming and requires additional resources. Tracheostomy in such situations is conventional and time-tested; however, it is not without complications; it needs special postoperative care, lengthens hospital stay, and also adds to expenses. ${ }^{4}$ Retromolar intubation may be an option, but the retromolar space may not be adequate in all adult patients. ${ }^{5}$

The submental intubation, first described by Altemir in 1986 has turned out to be an ingenious way of avoiding most of the problems associated with other forms of intubation. ${ }^{6}$ This technique avoids most of the problems associated with nasal intubation and tracheostomy. ${ }^{7}$ It is not time consuming, most of the post-operative complications associated with tracheostomy are avoided and it does not interfere with the surgical access. ${ }^{4}$ The scope of this technique has extended far beyond the realm of maxillofacial surgeries and it has been successfully used in orthognathic surgeries and elective aesthetic face surgeries as there is minimal distortion of the nasolabial soft tissue. ${ }^{7}$ It is also used in surgeries where both nasal and oral passages are used by the surgeons like oncological resection and reconstruction, oronasal fistula, selected cleft lip, and palate surgeries. Repair of congenital malformations, skull base surgery, multiple or complex facial osteotomies, transfacial oncologic procedures of the cranial base, and craniofacial surgeries are also current indications for submental intubation. ${ }^{8}$

\section{METHODOLOGY}

This retrospectivestudy wasperformed at maxillofacial surgery department of KRL General Hospital Islamabad, which is a level II trauma care center. Retrospective data was collected from electronic patient records and patient's operation notes from 1st June 2013 to 30th April 2018. All patients who had submental intubation performed irrespective of age and gender were selected. Intraoperative, post-operative course and complication details were extracted from the surgical and anesthesia notes. All patients were contacted during compilation of data and were asked to describe their perception of scar as "acceptable" or "unacceptable".

\section{Technique:}

All the procedures were performed as a team by the same anesthesiologist and the same maxillofacial surgeon. The cases were discussed and planned well in advance of surgery, including order of intubation, type of tube and task allocation. In all cases a tracheostomy set was made available in case of an inadvertent failure at any step. All of the submental intubations were done via a midline approach. The size of endotracheal tube was determined on the requirements for orotracheal intubation. An armored endotracheal cuffed tube with a detachable connector was used. The tongue was pulled upwards with a babcock forceps and a $1.5 \mathrm{~cm}$ vertical incision was marked at the base of anterior tongue. For extraoral incision, a $2 \mathrm{~cm}$ horizontal midline line was marked at the submental fold (Figure 1). The skin was prepped with $7.5 \%$ povidone iodine solution and draped. Lidocaine $2 \%$ with 1:100,000 adrenaline was infiltrated subcutaneously and intraorally. After successful oral intubation, a monopolar cautery with Colorado needle was used to make an incision intraorally. An incision was made extraorally with a scalpel along the marked line into the subcutaneous plane. A hemostat was used for blunt dissection through the mylohyoid. The curved hemostat was used again to join it to the extraoral incision (Figure 2). Adequate hemostasis was ensured. The hemostat was placed in position and the anesthesiologist was informed as being ready. The anesthesiologist then removed the tube connector, which was grasped by the hemostat (Figure 3), then fed the ETT through the incision extraorally. The connector was reapplied and the tube was connected to the anesthesia machine (Figure 4). The anesthesiologist then verified correct tube placement by auscultation and capnography, secured it with $2 / 0$ silk using Roman Garter technique. Intraorally, the tube was adjusted along the floor of the mouth lateral to the tongue base (Figure 5). 


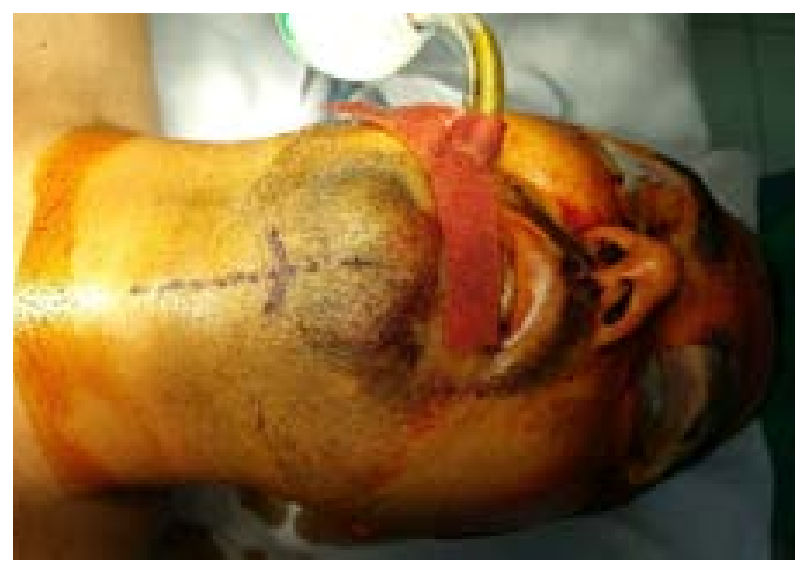

Figure 1: Marking of a midline extraoral incision at submental fold

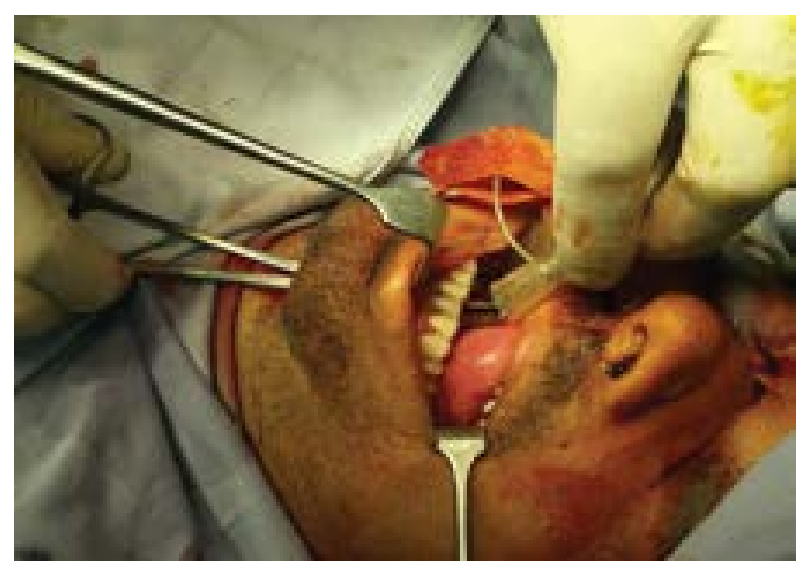

Figure 2: After blunt dissection to connect intra and extraoral incisions, the ETT is withdrawn and grasped by the hemostat

At the end of surgery, the tube was shifted back to the oral cavity to convert the submental intubation into a standard oral intubation. The intra- and extraoral incisions were closed. The extraoral sutures were removed after 7 - 10 days postoperatively.

\section{RESULTS}

During the period of study, a total of 570 patients with maxillofacial trauma of various degrees were attended at our tertiary care hospital. Out of these, 253 were operated under general anesthesia. From these operated patients, $185(73.1 \%)$ were males and $68(26.9 \%)$ were females; age range was $1.3-78$ years with mean of 32.7 years. A total of 7 submental intubations were performed. Out these, 2 patients had panfacial trauma, 3 had comminuted Lefort II and III fractures and 2 had comminuted nasoorbitoethmoid (NOE)/ frontal complex fractures. Two of these patients were provided overnight ventilator support after surgery, in which ETT was brought back to the

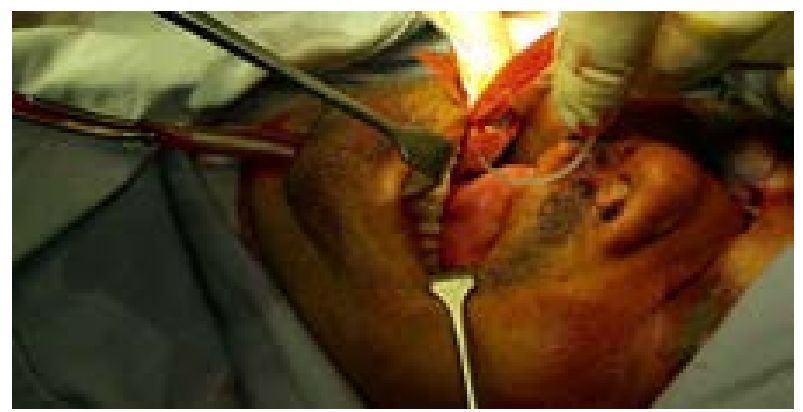

Figure 3: Pulling the ETT through the incision

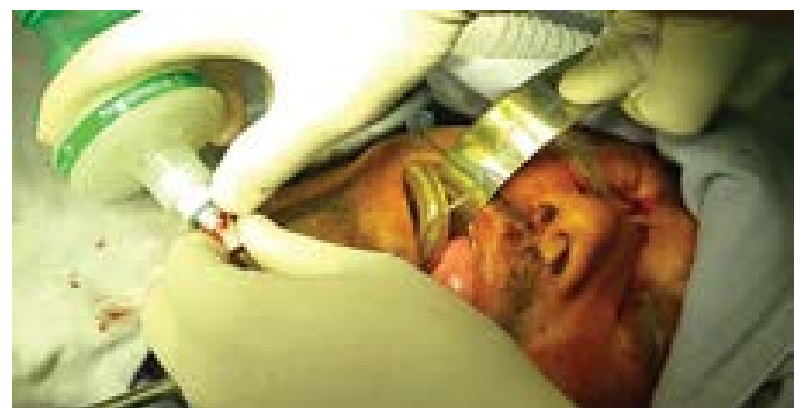

Figure 4: ETT is connected to the anesthesia machine and patency is confirmed with auscultation and capnography

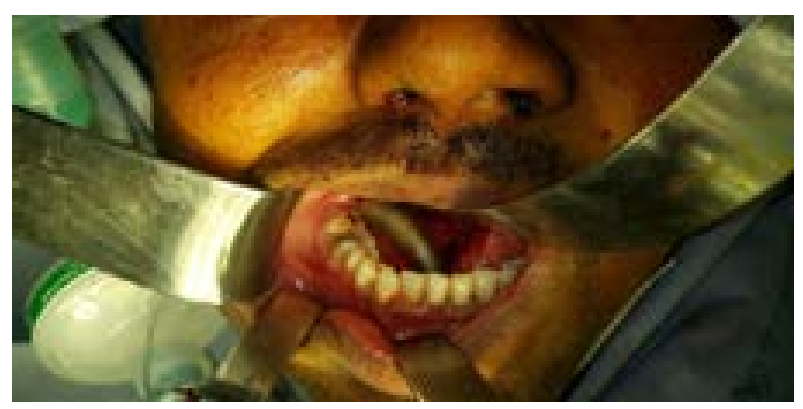

Figure 5: The tube is secured with $2 / 0$ silk and adjusted to lateral aspect of tongue to move it out of surgical field

oral cavity for postoperative airway management. The rest of the 5 patients were recovered after surgery and had an uneventful recovery. Postoperative esthetic concerns were negligible. One patient had persistent orocutaneous fistula with saliva leakage, which was managed conservatively and healed in 4 weeks post-surgery. Interestingly, this was the only patient in which an existing laceration was used which corresponded to certain extent with the intended incision design.

\section{DISCUSSION}

Management of airway is always a primary concern during any maxillofacial surgery. Implementing a safe and acceptable alternative to tracheostomy for short 
Table 1: Demographic details of patients along with type of fracture and complications

\begin{tabular}{c|c|c|c|c|c|c|c}
\hline S. No. & Age & Gender & $\begin{array}{c}\text { Type of } \\
\text { Fracture }\end{array}$ & Extubation & $\begin{array}{c}\text { Immediate } \\
\text { complications }\end{array}$ & $\begin{array}{c}\text { Delayed } \\
\text { Complications }\end{array}$ & $\begin{array}{c}\text { Patient's perception } \\
\text { of scar }\end{array}$ \\
\hline 1 & 34 & M & Lefort I//III & Immediate & None & None & Acceptable \\
\hline 2 & 27 & F & Panfacial & Immediate & None & None & Acceptable \\
\hline 3 & 41 & M & Lefort II/III & Immediate & None & None & Acceptable \\
\hline 4 & 43 & M & NOE/ Frontal & Day 1 postop & None & None & Acceptable \\
\hline 5 & 23 & M & Panfacial & Immediate & None & None & Acceptable \\
\hline 6 & 26 & F & NOE/ Frontal & Immediate & None & Salivary fistula & Acceptable \\
\hline 7 & 31 & M & Lefortll/III & Day 1 postop & None & None & Acceptable \\
\hline
\end{tabular}

term airway management is a desirable objective in management of complex facial trauma. ${ }^{1}$ Nasotracheal intubation is contraindicated in skull base fractures and nasal bone fractures. ${ }^{2}$ Tracheostomy has been a standard procedure in complex maxillofacial trauma but has been associated with some complications. Risk of tracheal stenosis, hemorrhage, and a residual ugly neck scar are few of the complications. It is therefore difficult to propose tracheostomy to patients suffering from an isolated facial trauma who will not require prolonged airway management. ${ }^{4}$

There have been several attempts to achieve short term airway management including retromolar intubation and nasal tube switch technique. Retromolar intubation has disadvantages of being more traumatic, obtrusive, costly and requiring more time. Nasal tube switch has been associated with extra time consumption and risk of aspiration due to post nasal bleeding. ${ }^{5,9}$ The present study is a retrospective analysis of the submental approach as an alternative to above mentioned techniques.

Altemir in 1986 utilized a lateral neck approach with a subperiosteal dissection on the lingual aspect of mandible. ${ }^{6,8}$ Since then, many individual modifications have been suggested by various surgeons. Most authors considered the Altemir's subperiosteal approach unnecessary and not essential. ${ }^{7}$ Other modifications included a strict midline approach. ${ }^{10}$ It offers multiple advantages; firstly it helps to hide the scar in submental fold, secondly the midline area between the mylohyoid bellies on each side is relatively avascular and so bleeds less and lastly, as compared to the lateral approach, there are no vital structures in the area that can be potentially damaged during dissection. ${ }^{11}$ These include the Stensen's duct, lingual nerve and medium diameter blood vessels. ${ }^{7,11}$

Since the midline approach does not carry the risk of damage to vital structures in the area, the most expected complication remains the postoperative infections. Caron et al, in a review of 25 patients with maxillofacial trauma reported 1 (4\%) patient with infection at the incision site. ${ }^{12}$ Anwer et al ${ }^{13}$ reported 2 of 14 (14\%) patients with postoperative superficial skin infections. We observed no significant postoperative infections at the incision site in any of our patients.

Another aspect to consider is the postoperative scar as an extraoral incision is involved. Since the submental area is concealed below the chin fold, and the propensity of the area to heal well leaves minimal scarring, generally the esthetic results are not of a major concern. All of our patients described their scars as 'acceptable' and not at all to barely visible. Meyer et a ${ }^{14}$ reported 1 (4\%) patient with hypertrophic scarring and $2(8 \%)$ patients with floor of mouth abscesses in their series of 25 patients. These complications were not seen in any of our patients.

There is still no consensus regarding superiority of one technique over another as a mode of securing airway in complex craniofacial injury repair. This may be true to the fact that there is no "one for all" solution to having an ideal airway. Paucity of published literature (case reports and case series) and quality of evidence limit definite recommendation on its use. Patient's ability to cooperate with the procedure, liaison between the surgeons and the anesthesiologists, experience of airway managers to deal with the situation, and benefits of single versus multiple surgical interventions are important considerations. Prolonged period of time is required for the adequate planning, preparation of the patient, personnel and procedure, which limits the utility of this technique in emergency situations.

\section{CONCLUSION}

In summary, submental intubation is a suitable option for intraoperative airway control in selected complex 
craniofacial injuries. This technique offers most of the advantages of both orotracheal and nasotracheal intubation at the same time.

Conflict of interest: None declared by the authors
Authors' contribution: All authors took part in the conduct of study, collection and analysis of the data and preparation of this manuscript.

Consent: Patient consent was obtained for use of pictures for academic purposes.

\section{REFERENCES}

1. Chandra Shekar BR, Reddy CV. A fiveyear retrospective statistical analysis of maxillofacial injuries in patients admitted and treated at two hospitals of Mysore city. Indian J Dent Res. 2008;19:304-8. [PubMed] [Free full text]

2. Nwoku AL, Al-Balawi SA, Al-Zahrani SA. A modified method of submental oroendotracheal intubation. Saudi Med J. 2002;23:73-6. [PubMed]

3. Arrowsmith JE, Robertshaw HJ, Boyd JD. Nasotracheal intubation in the presence of frontobasal skullfracture. Can J Anaesth. 1998;45:71-5. [PubMed]

4. Durbin CG., Jr Early complications of tracheostomy. Respir Care. 2005;50:511-5. [PubMed]

5. Xue FS, He N, Liao X, Xu XZ, Liu JH. Further observations on retromolar fibreoptic orotracheal intubation in patients with severe trismus. Can J Anaesth. 2011;58:868-9. [PubMed] [Free full text]

6. Altemir FH. The submental route for endotracheal intubation: A new technique. J Maxillofac Surg.1986;14:64-5. [PubMed]

7. Jundt JS, Cattano D, Hagberg CA, Wilson JW. Submental intubation: A literature review. Int J OralMaxillofac Surg. 2012;41:46-54. [PubMed] [Free full text]

8. Altemir $\mathrm{FH}$, Montero $\mathrm{SH}$. The submental route revisited using the laryngeal mask airway: A technicalnote. J Craniomaxillofac Surg. 2000;28:343-4. [PubMed]

9. Xue FS, He N, Liao X, Xu XZ, Liu JH. Further observations on retromolarfibreopticorotracheal intubation in patients with severe trismus. Can J Anaesth. 2011;58:8689. [PubMed]

10. Biglioli F, Mortini P, Goisis M, Bardazzi A, Boari N. Submental orotracheal intubation: An alternativeto tracheostomy in transfacial cranial base surgery. Skull Base. 2003;13:189-95. [Free full text]

11. Kar C, Mukherjee S. Submental intubation: An alternative and cost-effective technique for complexmaxillofacial surgeries. J Maxillofac Oral Surg. 2010;9:266-9. [PubMed] [Free full text]

12. Caron G, Paquin R, Lessard MR, Trepanier CA, Landry PE. Submental endotracheal intubation: An alternative to tracheotomy in patients with midfacial and panfacial fractures. J Trauma Infect Crit Care.2000;48:23540. [PubMed]

13. Anwer HM, Zeitoum IM, Shehata EA. Submandibular approach for tracheal intubation in patients with panfacial fractures. $\mathrm{Br} \mathrm{J}$ Anaesth. 2007;98:835-40. [PubMed] [Free full text]

14. Meyer C, Valfrey J, Kjartansdorttir T, Wilk A, Barriere P. Indication for and technical refinements ofsubmental intubation in oral and maxillofacial surgery. J Cranio-Maxillofac Surg. 2003;31:383-8. 